\title{
Mesenteric Fibromatosis of the Small Bowel Mesentery After Gastric Bypass Surgery
}

\author{
Yanira Perez, MD, MIS Fellow, John D. Webber, MD, FACS, David Edelman, MD, FACS \\ Wayne State University, Detroit, MI, USA (all authors).
}

\begin{abstract}
Mesenteric fibromatosis poses a diagnostic and therapeutic challenge. We report a case of giant cell mesenteric fibromatosis tumor arising from the mesentery of the small intestine in a patient two years after a Roux-en-Y gastric bypass. A 47-year-old African-American female presented as a transfer from an outside institution with a large abdominal mass that was initially diagnosed as an intraabdominal cyst on computed tomography scan. The tumor was successfully excised surgically and the diagnosis of mesenteric fibromatosis tumor was confirmed on immunohistochemical analysis. To our knowledge, this is the only reported case of mesenteric fibromatosis tumor arising from the jejunojejunostomy anastomosis of the small bowel mesentery after Roux-en-Y gastric bypass surgery to treat morbid obesity.
\end{abstract}

Key Words: Mesenteric fibromatosis, Small bowel mesentery, Gastric bypass.

Citation Perez Y, Webber JD, Edelman D. Mesenteric fibromatosis of the small bowel mesentery after gastric bypass surgery: a case report and review of literature. CRSLS e2013.00264. DOI: 10.4293/CRSLS.2013.00264.

Copyright (c) 2015 by SLS, Society of Laparoendoscopic Surgeons. This is an open-access article distributed under the terms of the Creative Commons Attribution-Noncommercial-ShareAlike 3.0 Unported license, which permits unrestricted noncommercial use, distribution, and reproduction in any medium, provided the original author and source are credited.

Address correspondence to: David A. Edelman, MD, Wayne State University School of Medicine, Department of Surgery, 3990 John R. Street, Detroit, MI 48201. E-mail address: dedelman@med.wayne.edu

\section{INTRODUCTION}

Mesentric fibromatosis is a rare, deep-seated, usually unencapsulated benign proliferation of fascial or musculoaponeurotic structures that tend to recur without distant metastasis. ${ }^{1,2}$ Although fibromatosis tumors are considered benign, they are locally aggressive with a high propensity for infiltration of adjacent tissues. ${ }^{3}$ Data concerning these tumors as a distinct pathological entity are lacking. ${ }^{4}$ The etiology of mesenteric fibromatosis is yet to be determined. Most reported cases have been in association with Gardner syndrome, previous trauma, and prolonged estrogen intake. ${ }^{5}$ Mesenteric fibromatosis can occur as a primary condition in the absence of any predisposing condition. The tumor presents either as a result of mass effect or from obstruction of the surrounding structures. Most intraabdominal fibromatosis tumors arise in the mesentery, particularly in the small bowel, and are the most common primary tumor of the mesentery. ${ }^{6-8}$ The present report is a rare case of giant cell mesenteric fibromatosis tumor arising from the jejunojejunostomy anastomosis of the small bowel mesentery in a patient presenting with abdominal pain, fatigue syndrome, status post Roux-en-Y gastric bypass 18 months previously.

\section{CASE REPORT}

A 47-year-old African-American female, with an initial body mass index of 63.7, status post laparoscopic Rouxen-Y gastric bypass 18 months prior presented with abdominal pain and discomfort that had been gradually increasing over a two-month period. The pain was felt over her right upper quadrant, described as an 8 out of 10 in severity, and nonradiating. She described symptoms of nausea, early satiety, anorexia, abdominal distention, and worsening constipation. She had lost 211 pounds since her bariatric surgery, resulting in a lower body mass index of 28.6. She denied any recent weight loss. She described persistent fatigue for the last several months. No family members were previously diagnosed with gastrointestinal cancer. On clinical examination, there was a large solid mass palpable in the right upper quadrant of the abdomen. It was nonpulsatile, immobile, and tender to deep palpation. Her routine blood results were within normal limits. Computerized tomography scan of the abdomen and pelvis were obtained.

The abdominal scan demonstrated a large right upper abdominal mass with heterogeneous density, measuring $18 \times 14 \times 14 \mathrm{~cm}$ (Figure 1). The mass was well circum- 
Mesenteric Fibromatosis of the Small Bowel Mesentery After Gastric Bypass Surgery: A Case Report and Review of Literature, Perez Y et al.
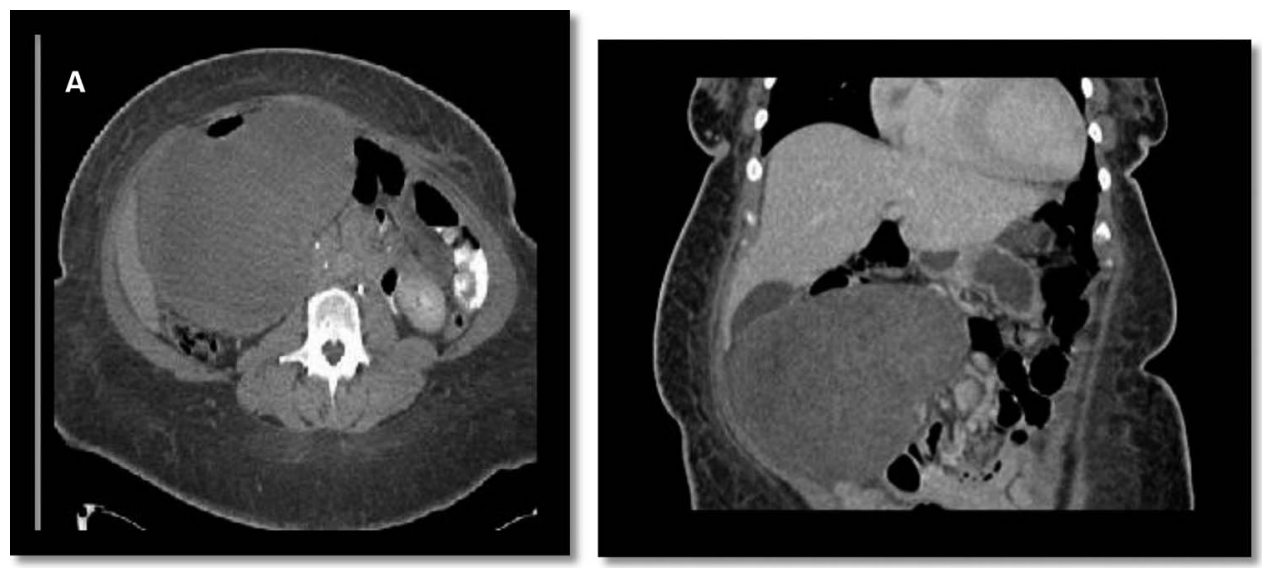

Figure 1. Contrast enhanced tomography scan showing a large inhomogeneous mass-occupying lesion. A, Axial section; B, sagittal section.

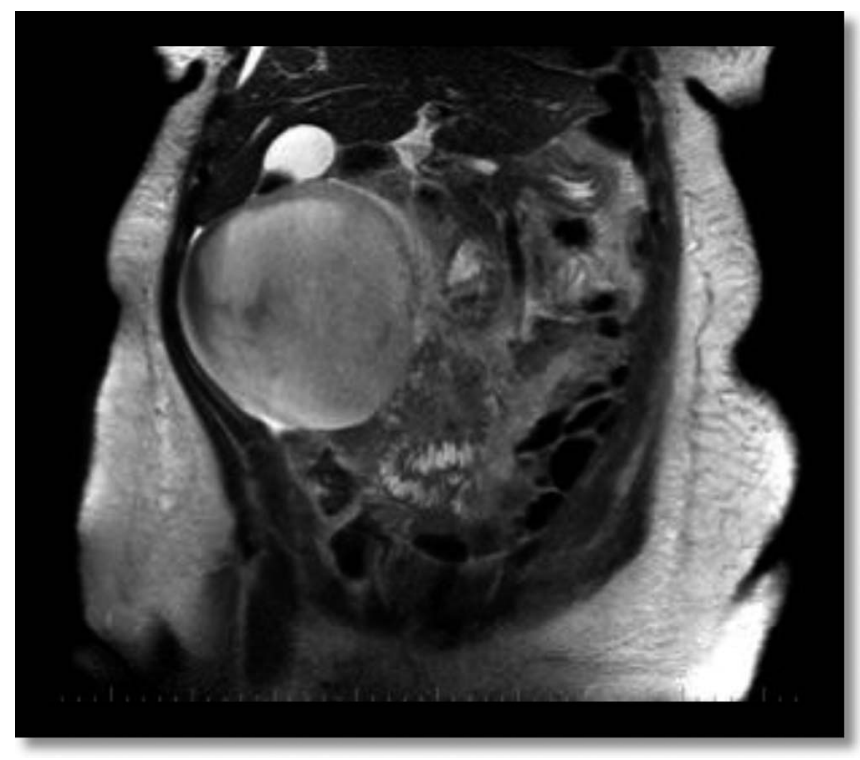

Figure 2. Magnetic resonance imaging of the abdomen and pelvis. T2-weighted image showing a large, intraperitoneal, welldefined mass displacing the liver and bowels with heterogeneous bright and low signals.

scribed with mass effect on nearby structures. There was no evidence of metastasis to the adjacent viscera and peritoneal cavity. To further characterize the mass, a magnetic resonance imaging scan was obtained (Figure 2). Imaging studies were highly suggestive of a cystic structure.

A diagnostic laparoscopy was initially performed, followed by a midline incision exploratory laparotomy because of the uncertain nature of the mass. A large, rounded, and well-demarcated mass (Figure 2) arising from the mesentery of the jejunum at the jejunojejunostomy anastomosis from the patient's Roux-en-Y gastric bypass procedure was visualized. The jejunum was adherent to the mass. There were prominent vessels feeding into the tumor from the mesentery. The rest of the abdomen was unremarkable. The tumor was resected en bloc and sent for histopathological analysis. The pancreaticobiliary limb and the Roux-en-Y limb were left in discontinuity from the common channel after the mass was excised. The jejunojejunostomy anastomosis was recreated using a linear Endo-GIA stapler (Covidien, Mansfield, Massachusetts). The pancreaticobiliary limb was anastomosed proximally to the common channel, whereas the Roux-en-Y limb was anastomosed $10 \mathrm{~cm}$ down on the common channel (Figure 3). Postoperatively, the patient made an uneventful recovery and was discharged home on postoperative day 5 .

On gross pathologic examination, the specimen consisted of the mass with partial small bowel tissue (Figure 4). The specimen weighed $1740 \mathrm{~g}$, with the tumor measuring $19 \times$ $15.3 \times 12.4 \mathrm{~cm}$. The surface of the tumor was smooth. Serial sectioning of the mass revealed a fleshy, firm, tanyellow mass with focal hemorrhage without necrosis. The mucosal margins were found to be negative for involvement.

Microscopic examination indicated that the mass was paucicellular and composed of interlacing spindle cells with minimal atypia. Mitoses and necrosis were not identified. Immunohistochemistry demonstrated the cells to be diffusely positive for $\beta$-catenin and vimentin. Furthermore, staining for CD 117 (c-KIT), CD 34, desmin, actin, 


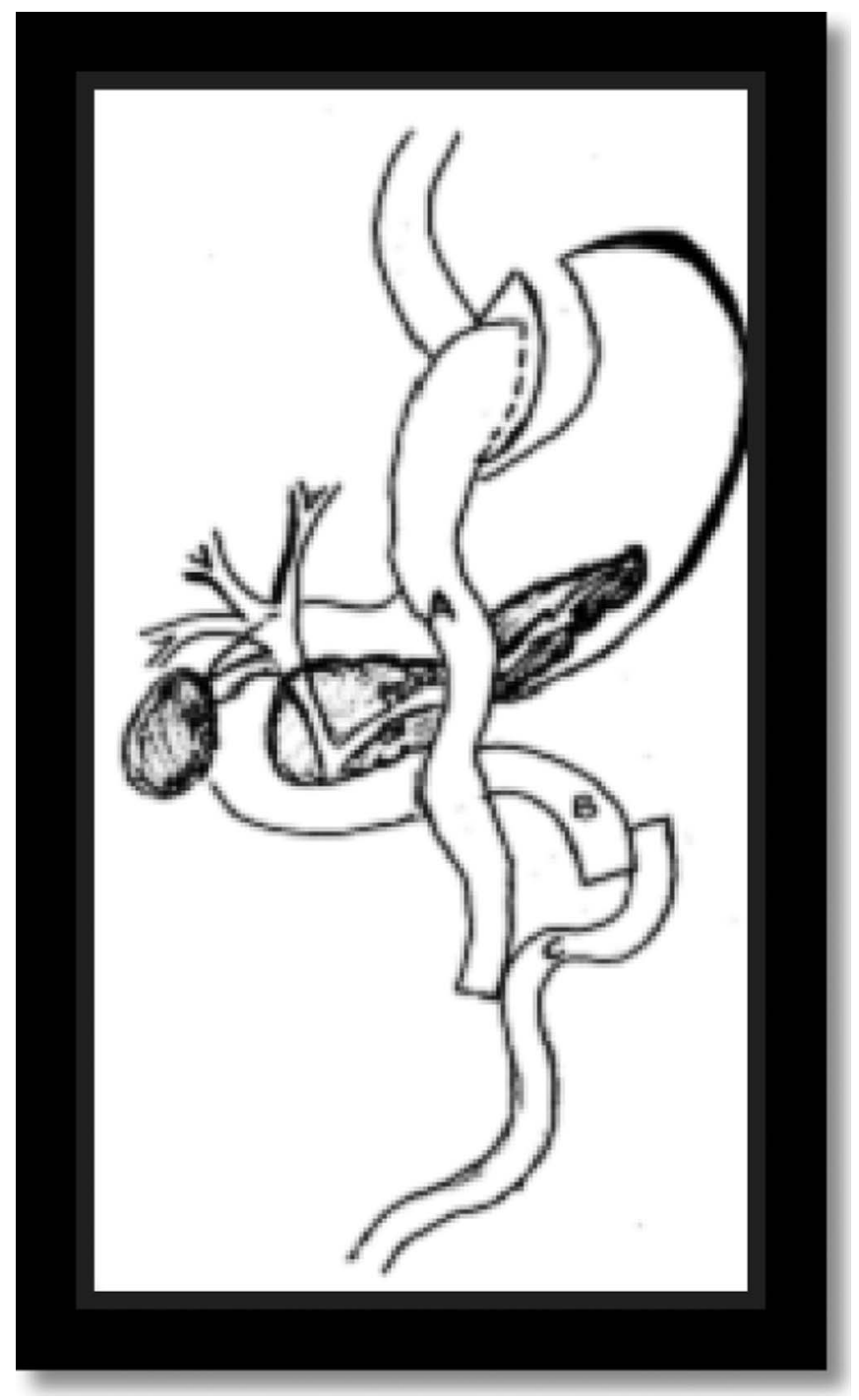

Figure 3. Schematic of revised jejunojejunostomy anastomosis. A, Roux-en-Y limb; B, pancreaticobiliary limb; C, common channel.

ALK-1, and S-100 were negative. Thus, the morphology and immunoprofile supported the diagnosis of mesenteric fibromatosis.

\section{DISCUSSION}

The concept of fibromatosis as a group of similar lesions was first coined by Stout in 1961. ${ }^{9}$ The fibromatosis can affect both superficial and deep parts of the body. Superficial fibromatosis involves the face and neck (fibromatosis coli), palms (Dupuytren contracture), feet (Ledderhose disease), penis (Peyronie dis- ease), shoulder, thigh, buttock, and trunk. ${ }^{10}$ The deep variant involves the abdominal wall, mesentery, retroperitoneum, mediastinum, and abdominal cavity. ${ }^{11,12}$ Fibromatosis tumors are rare, accounting for $0.03 \%$ of all tumors. ${ }^{13}$ Mesenteric fibromatosis tumors are most frequently found in young adults with a peak incidence at 30 years of age. The sex incidence varies, with some reporting an equal frequency in both males and females, whereas others documented a female predominance only. ${ }^{14,15}$ Most patients with mesenteric fibromatosis tumors remain clinically asymptomatic, with little or no focal symptoms until late in their course, at which stage they complain of abdominal pain and discomfort, as well as organ compression symptoms. Reported complications of intraabdominal fibromatosis tumors include ureteric obstruction, intestinal obstruction, intestinal ischemia, deterioration in an ileoanal anastomosis, abscess, and hepatic pneumatosis. ${ }^{16-21}$

The diagnosis of a fibromatosis tumor can only be established histologically. Our patient's findings reflected these characteristics. However, various mesenchymal tumors in the gastrointestinal tract or abdominal cavity may have similar gross appearance, histology, and immunophenotype. $^{22}$ In our patient, negative results of staining for desmin, smooth muscle actin, and S-100 excluded other mesenchymal tumors such as fibrosarcoma, leiomyoma, leiomyosarcoma, and malignant peripheral nerve sheath tumor. The clinical, radiological, and gross appearance of the specimen was most suggestive of gastrointestinal stromal tumor or desmoid. But as was noted in the case report, the lack of staining for CD 117 by immunohistochemistry and the absence of a c-KIT gene mutation were helpful in ruling out the former. ${ }^{23,24}$ Furthermore, the $\beta$-catenin gene mutation confirmed the diagnosis of fibromatosis tumor.

Despite complete resection with negative microscopic margins, fibromatosis tumors have recurrence rates as high as $40 \% .6,25,26$ Therefore, it is recommended that surgery should be performed only when absolutely necessary. ${ }^{27}$ This was the case with our patient who had a potentially life-threatening complication of intestinal obstruction secondary to the tumor's mass effect. She has remained recurrence free for 1 year. There is no standardized protocol for follow-up of patients with fibromatosis tumors. Currently, there is no surveillance protocol or serological marker to detect or diagnose fibromatosis tumors at earlier stages. 

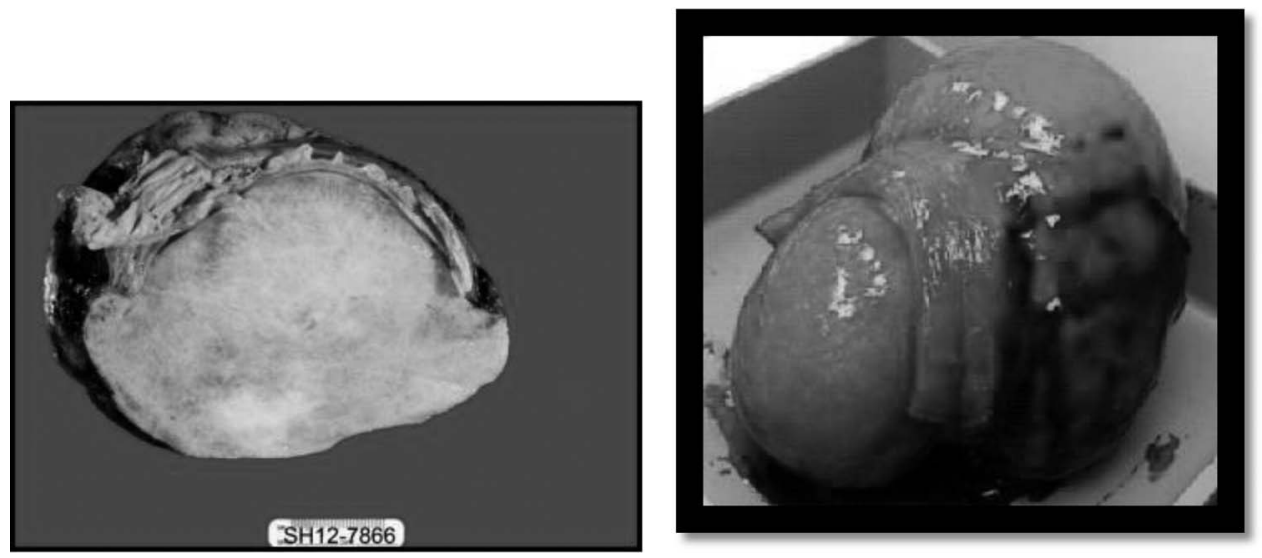

Figure 4. Large, rounded, well-demarcated mass. The gross measurement of the mass was $19 \times 15.3 \times 12.4 \mathrm{~cm}$.

In conclusion, we report a case of a fibromatosis tumor originating from a jejunojejunostomy anastomosis after a Roux-en-Y gastric bypass procedure. Despite the unusual location, both histology and genetic studies helped confirm the diagnosis.

\section{References:}

1. Yang CH, Sheen-Chen SM, Lu CC, Ko SF, Eng HL. Computed tomographic presentation of mesenteric fibromatosis. Dig Dis Sci. 2005;50:348-350.

2. Enzinger FM, Weiss SW. Fibromatosis. In: Enzinger FM, Weiss SW, editors. Soft Tissue Tumor. St. Louis, MO: C.V. Mosby Co.;1995: 201-229.

3. Lahat G, Nachmany I, Itzkowitz E, et al. Surgery for sporadic abdominal desmoid tumor: is low/no recurrence an achievable goal? Isr Med Assoc J. 2009;11:398-402.

4. McCormack D, Kesha K, Tittle SL, Saldinger PF. Mesenteric fibromatosis mimicking a gastrointestinal stromal tumor. Conn Med. 2010;74:197-200.

5. Karakousis CP, Berjian RA, Lopez R, Rao U. Mesenteric fibromatosis in Gardner's syndrome. Arch Surg. 1978;113:9981000 .

6. Shields CJ, Winter DC, Kirwan WO, Redmond HP. Desmoid tumours. Eur J Surg Oncol. 2001;27:701-706.

7. Einstein DM, Tagliabue JR, Desai RK. Abdominal desmoids: CT findings in 25 patients. AJR Am J Roentgenol. 1991;157:275279.

8. Burke AP, Sobin LH, Shekitka KM, Federspiel BH, Helwig EB. Intra-abdominal fibromatosis. A pathologic analysis of 130 tumors with comparison of clinical subgroups. Am J Surg Pathol. 1990;14:335-341.

9. Stout AP. The fibromatosis. Clin Orthop. 1961;19:11-18.
10. Faria SC, Iyer RB, Rashid A, Ellis L, Whitman GJ. Desmoid tumor of the small bowel and the mesentery. AJR Am J Roentgenol. 2004;183:118.

11. Lath C, Khanna PC, Gadewar SB, Agrawal D. Inoperable aggressive mesenteric fibromatosis with ureteric fistula. Case report and literature review. Eur J Radiol. 2006;59:117-121.

12. Kabra V, Chaturvedi P, Pathak KA, deSouza LJ. Mesenteric fibromatosis: a report of three cases and literature review. Indian J Cancer. 2001;38:133-136.

13. Guglielmi G, Cifaratti A, Scalzo G, Magarelli N. Imaging of superficial and deep fibromatosis. Radiol Med. 2009;114:12921307.

14. Yannopoulos K, Stout AP. Primary solid tumors of the mesentery. Cancer. 1963;16:914-927.

15. Dong-Heup K, Kim DH, Goldsmith HS, Quan SH, Huvos AG. Intra-abdominal desmoid tumor. Cancer. 1971;27:10411045.

16. Clark SK, Neale KF, Landgrebe JC, Phillips RK. Desmoid tumours complicating familial adenomatous polyposis. BrJ Surg. 1999;86:1185-1189.

17. Church JM. Mucosal ischemia caused by desmoid tumors in patients with familial adenomatous polyposis: report of four cases. Dis Colon Rectum. 1998;41:661-663.

18. Sagar PM, Moslein G, Dozois RR. Management of desmoids tumors in patients after ileal pouch-anal anastomosis for familial adenomatous polyposis. Dis Colon Rectum. 1998;41:1350-1355; discussion 1355-1356.

19. Penna C, Tiret E, Parc R, et al. Operation and abdominal desmoid tumors in familial adenomatous polyposis. Surg Gynecol Obstet. 1993;177:263-268.

20. Cholongitas E, Koulenti D, Panetsos G, et al. Desmoid tumor presenting as intra-abdominal abscess. Dig Dis Sci. 2006;51:68-69. 
21. Prat A, Peralta S, Cuellar H, Ocana A. Hepatic pneumatosis as a complication of an abdominal desmoid tumor. J Clin Oncol. 2007;25:897-898.

22. Abraham SC. Distinguishing gastrointestinal stromal tumors from their mimics: an update. Adv Anat Pathol. 2007; $14: 178-188$.

23. Rakha EA, Kandil MA, El-Santawe MG. Gigantic recurrent abdominal desmoid tumour: a case report. Hernia. 2007;11: 193-197.

24. Hailemariam S, Jaeger P, Goebel N, Grant JW. Mesenteric fibromatosis with ureteric stenosis. Postgrad Med J. 1988;64: $79-81$.
25. Nuyttens JJ, Rust PF, Thomas CR Jr, Turrisi AT 3rd. Surgery versus radiation therapy for patients with aggressive fibromatosis or desmoid tumors: a comparative review of 22 articles. Cancer. 2000;88:1517-1523.

26. Rampone B, Pedrazzani C, Marrelli D, Pinto E, Roviello F. Updates on abdominal desmoid tumors. World J Gastroenterol. 2007;3:5985-5988.

27. Ballo MT, Zagars GK, Pollack A, Pisters PW, Pollack RA. Desmoid tumor: prognostic factors and outcome after surgery, radiation therapy, or combined surgery and radiation therapy. J Clin Oncol. 1999;17:158-167. 